\title{
Illumination Estimation for Nature Preserving Low-Light Image Enhancement
}

\author{
Kavinder Singh* and Anil Singh Parihar ${ }^{\dagger}$, Member, IEEE
}

\begin{abstract}
Low-light images may degrade the performance of a vision-based system due to low visibility. Thus, enhancement of low-light images is an essential step to achieve the effective object and feature extraction from low-light images. In this paper, we present a new approach to estimate illumination and reflectance for low-light image enhancement. The proposed algorithm performs a structure-aware estimation of the initial illumination to preserve natural variation in the illumination of the images. The work formulates a multi-objective optimization function using textural and structural details of the image. It refines the illumination estimation by minimizing the multi-objective function. The proposed algorithm performs image enhancement with color constancy and preserves the natural details of the image. To analyze the performance of the proposed approach, we perform qualitative and quantitative analysis on low-light images with varying illumination from several benchmark datasets. The performance comparison with state-of-the-art algorithms shows the superiority of the proposed algorithm. Moreover, the proposed method performs favorably for low-light images with varying illumination while enhancing the details of the dark regions.
\end{abstract}

Index Terms-Low-light image, illumination, illumination estimation, image enhancement.

\section{INTRODUCTION}

$\mathbf{I}$ MAGES with proper illumination are always desired in the design of any vision-based system. However, many times the environment is not conducive to capture an image with proper illumination and results in a low-light image. Moreover, the other cause of low-light images may be improper or variable lighting in a scene, night time imaging, improper camera setting, or limitations with the camera, bad lighting sources, etc. Low-light images suffer from low visibility of object details due to inadequate illumination. Sometimes an image may contain two types of regions: regions with properly illuminated objects, and regions with poorly illuminated objects(i.e., image with varying illumination). Lowlight images pose challenges in object and feature detection and may degrade the performance of a vision-based system. Low-light image enhancement may improve the visual quality of an image. Thus, results in gathering more information about the scene details. However, an inferior enhancement algorithm may result in loss of crucial details and introduce undesirable artifacts. Thus, designing an effective algorithm for the enhancement of low-light images is a challenging task.

In literature, many researchers have attempted to develop algorithms for low-light image enhancement. Low-light images contain most of the pixels with low intensity and can

Kavinder Singh* and Anil Singh Parihar ${ }^{\dagger}$ are with the Department of Computer Science \&. Engineering, Delhi Technological University, New Delhi, INDIA (email: *kavinder85@gmail.com, and † parihar.anil@gmail.com). be loosely considered as low contrast images. To this end, one of the simplest and fundamental approaches is Histogram Equalization (HE) [1]. HE based approaches focus on performing intensity transformation using frequencies of pixel intensities. However, HE based methods may introduce saturation effect, over or under enhancement, halo effects, loss of local details, etc. Several modifications of HE are developed to overcome these drawbacks. Abdullah et al. [2] proposed a dynamic HE (DHE) algorithm by dividing a histogram at local minima and keeping the dynamic range of each subhistogram in proportion to the original image. Parihar and Verma [3] developed an entropy-based DHE and proposed an optimal division of histogram using entropy. Many algorithms $[4,5,6]$ use a $2 \mathrm{D}$ histogram to incorporate local characteristics of the image to retain its natural characteristics. Parihar et al. [7] proposed an algorithm Fuzzy Contextual Contrast Enhancement (FCCE). FCCE uses fuzzy properties of the images to develop a fuzzy difference histogram (FDH). The authors further proposed a new intensity transfer function based on the intensity and local characteristics of the image to achieve natural-looking enhanced images. The above algorithms give reasonably good performance in properly illuminated regions. However, these algorithms focus on contrast enhancement rather than improving the illumination of the image, thus sometimes fail to improve the contrast of low-light images and introduce artifacts like saturation effect, halo effect, etc.

It has been found that Retinex[8] based enhancement algorithms work reasonably well for low-light images. In the Retinex theory, an image is considered as the product of illumination and reflectance. In early Retinex based approaches[9, 10, 11], lightness is considered as the ration of a pixel value to the average value of surrounding pixels and term it as center/surround operation. Jobson et al. [12] explored the properties of center/surround operation and used a Gaussian filter to develop Single Scale Retinex (SSR) for image enhancement. SSR has a major drawback that it provides either dynamic range compression or color constancy but fails to provide both simultaneously. To overcome these limitations, Jobson et al. [13] used multiple scales of Gaussian and developed Multi-Scale Retinex (MSR). MSR works well for grayscale images, but for color images, it may lead to unnatural color appearance. The problem of color restoration is attempted in another work by Jobson et al. [14]. In these algorithms $[12,13,14]$ reflectance is considered as the final enhanced image, which may look unnatural at times.

Many authors [15, 16, 17] have attempted simultaneous estimation of illumination and reflectance. It is an ill-posed problem to estimate reflectance and illumination from a single 
image. Fu et al. [15] proposed a method for computing both components from a single image simultaneously with probabilistic based prior (PIRE). The authors framed priors for both illuminance and reflectance to formulate maximum a posteriori (MAP). Fu et al. [16] proposed another approach for finding both components (i.e., illuminance and reflectance) from a single image based on the log of the Retinex model. These algorithms $[15,16]$ work well for objects with proper illumination in a low-light image, but for darker regions enhancement is limited. Li et al. [17] proposed an algorithm based on simultaneous estimation of reflectance and illumination. The work focuses on the inherent noise present in dark regions of the image. The algorithm gives good color constancy and enhancement. However, algorithm[17] results in degraded images due to over smoothening in many images. In general, the complexity of algorithms estimating both illumination and reflectance is high. Thus, these algorithms may not be useful for real-time applications.

Another set of algorithms [18, 19] estimate the illumination component and use it to find the reflectance component. The final enhanced image is obtained as the product of refined illumination and reflectance. Wang et al. [18] proposed an algorithm Natualness Preserved Enhancement Algorithm (NPEA) to deal with non-uniform illumination images. NPEA estimates illumination using a bright pass filter to find the reflectance component of the image. NPEA preserves naturalness at the cost of illumination improvement. Thus, darker regions may not enhance adequately. Fu et al. [19] proposed a fusion-based method to deal with weakly illuminated images. The algorithm uses a multi-fusion of Gaussian and Laplacian at multiple scales. The algorithm performs well in normal dark images but fails to provide significant lightness for a darker region. Guo et al. [20] proposed a low-light image enhancement algorithm using illumination map estimation (LIME). Similar to many other approaches, [18, 19] LIME estimates initial illumination by taking a pixel-wise maximum of all color channels of the low-light image. The algorithm computes refined illumination by optimizing a multi-objective problem based on initial illumination and its gradient. The performance of LIME is significantly good, however over enhancement occurs in properly illuminated regions of low-light images. Moreover, LIME sometimes fails to retain color constancy.

Estimation of illumination and reflectance is an ill-posed problem, and developing an effective low-light enhancement algorithm is still a challenging task. Most of the low-light image enhancement algorithms suffer from one or more of the following limitations:

- Inadequate enhancement of non-uniformly illuminated images, i.e., over-enhancement in the bright regions and under-enhancement in dark regions.

- Enhanced images with unnatural colors, i.e., Fail to preserve natural colors.

- Generation of undesired artifacts in the enhanced images.

- Unable to restrict the dispersion effect in light dominant regions of the low-light image with varying illumination.

- Loss of finer details in the resulting images.

- Trade-off between lightness enhancement and naturalness preservation, i.e. if an algorithm preserves naturalness, then the overall lightness of the image is low and vicea-versa.

In this paper, we proposed a new low-light image enhancement approach to overcome the above limitations. The proposed algorithm is named as Nature Preserving Lowlight Image Enhancement (NPLIE). NPLIE estimates initial illumination and performs optimal refinement. The proposed algorithm computes the reflectance component through an element-wise division of input image by illumination. The enhanced image is obtained as a product of adjusted illumination and reflectance component. In this work, we estimate initial illuminance from structure-aware smoothening of a low-light image using guided filters of variable box sizes. We compute refined illumination by solving the proposed multi-objective optimization problem. The major contributions of this work are as follows:

- Proposed a new method for estimation of initial illumination using Guided filters of variable and optimal box sizes.

- Formulated a multi-objective optimization problem with a new regularization term to preserve structural details in refined illumination.

- Proposed a solution of a multi-objective optimization problem using ADMM with the augmented lagrangian multiplier.

- Proposed an algorithm for low-light image enhancement and addressed all the limitations listed earlier.

The remaining paper is organized as follows. Section II contains a brief discussion about methodology. Section III presents results and analysis. Section IV concludes the proposed approach and findings.

\section{NATURE PRESERVING LOW-LIGHT IMAGE ENHANCEMENT (NPLIE)}

In this section, we present the proposed Nature Preserving Low-Light Enhancement (NPLIE) Algorithm. We discuss motivation in the first subsection and describe the detailed algorithm in the following subsections.

\section{A. Motivation}

In this work, we consider widely used Retinex model [10] of image formation, defined as:

$$
\mathbf{J}=\mathbf{R} \circ \mathbf{I}
$$

where $\mathbf{J}$ is an image, $\mathbf{R}$ is reflectance of the image, $\mathbf{I}$ is illumination, and symbol ' $\circ$ ' represents element-wise multiplication. The reflectance component depends on the objects in images and their colors. The illumination component depends on the source of light present at the time of image acquisition. The source of light might be sunlight, moonlight, an artificial light source like LED, CFL, camera flashlight, etc. Change in the source of light may lead to a change in the properties of illumination. Illumination properties of low-light images are different from day-light images. It is mainly because sunlight provides uniform illumination except in shaded regions. Generally, illumination in low-light images is non-uniform due to 
the dominance of light sources in some regions of the scene. In many algorithms $[18,19,20]$, illumination estimation is obtained from the image, and reflectance is computed using relation in (1). Thus, illumination estimation is a crucial step in the low-light image enhancement algorithm. Improper estimation of illumination leads to a degraded image and may produce artifacts.

In early works [12, 13], a smooth image is considered as an estimation of illumination. However, direct smoothening using a center/surround function does not capture the characteristics of non-uniform illumination. In dark regions, most of the information about illumination is available on the channel with the maximum value of intensity. Image with nonuniform illumination can have maximum illumination across any color channel depending on illumination for that particular region. Illumination does not depend on the color properties of the object. Many algorithms [18, 19, 20] use pixel-wise maximum across all color channels (bright-channel) as initial illumination. However, the bright-channel of low-light images may not represent true illumination. An image is indeed a $2 \mathrm{D}$ representation of $3 \mathrm{D}$ scenes (real world). Therefore, two adjacent objects in an image may be at far distant in reality, and illumination in such cases may also vary. However, maximum intensity for two adjacent objects may be similar, and bright-channel will not distinguish their illumination. Moreover, in light source dominated areas (artificial source of light) of low-light images, the bright-channel will scatter light in adjacent areas. Consider images shown in Fig. 1, it shows a portion dark image, illumination estimation, and enhanced image by LIME [20] algorithm and proposed algorithm. LIME algorithm considers bright-channel as initial illumination. The proposed algorithm (NPLIE) proposes a structure-aware initial illumination instead of bright-channel, described in section-II B. One may notice in the red box areas of Fig. 1 that structural details are lost in Fig. 1(d) (in case of LIME), while details are preserved in Fig 1(e) (in case of NPLIE). Similarly, in the green box of Fig. 1(d) artifacts are prominently visible
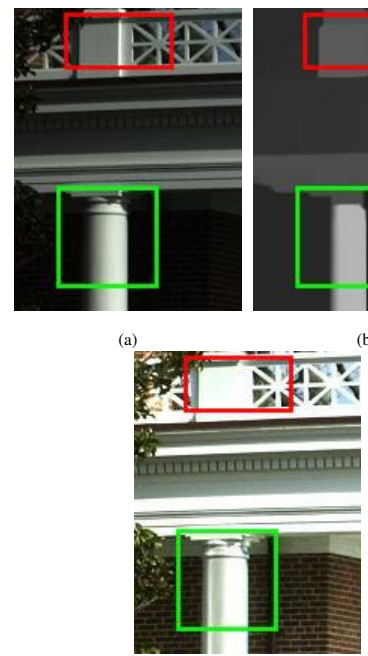

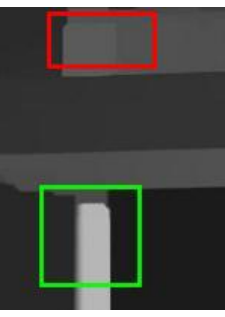

(b)
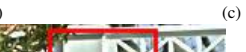

(c)
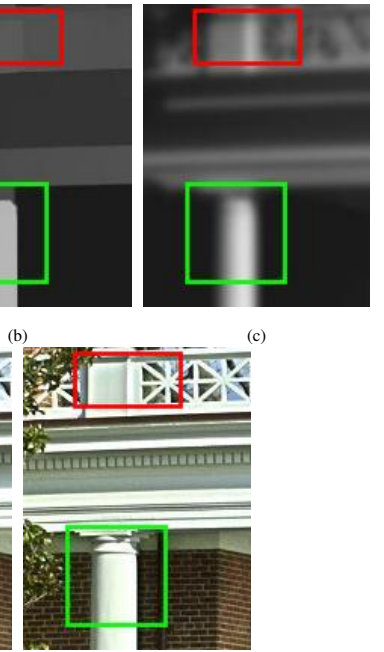

(e)

Fig. 1: Results of House. (a) Original Image; (b) Illumination of LIME; (c) Illumination of Proposed method; (d) LIME[20]; (e) Proposed Method. on the pillar. These losses of structural details and artifacts can be understood by analyzing the illumination estimation shown in Fig. 1(b). It may be noticed that illumination is changing abruptly, which is quite unnatural. Thus, in the case of images with non-uniform light conditions, a structure-aware illumination estimation is highly desirable. In this work, we proposed a structure-aware illumination estimation approach. To estimate non-uniform illumination, we apply guided filters with three different sizes of box-filter in each color channel. The algorithm preserves structural information in illumination by using multiple box sizes. We consider a pixel-wise maximum of structure-aware smoothen image as initial illumination estimation. We refine illumination by solving a multi-objective optimization problem. Section II B describes the proposed approach of illumination estimation in detail.

\section{B. Illumination Estimation for Nature Preserving Low-Light Image Enhancement}

Initial illumination has a defining effect on final illumination. Thus, it should be estimated carefully. The estimated illumination component should represent natural variation in illumination as per structural information of low-light images. The textural details of the image have almost no effect on the spatial variation of the illumination. Thus, illumination of an image may be considered to be independent of textural details. The Reflectance component preserves the textural details of the image. The algorithm should smoothen textural details while preserving structural details to achieve an actual (or near actual) estimation of the illumination. In the proposed approach, we first apply Guided filters [21] at three different box sizes to perform structure-preserving smoothening of textural detail at multiple scales. We apply guided filters to each channel with the same three box sizes. The proposed algorithm considers three box sizes: minimum box size $b_{\min }=3$, maximum box size $b_{\max }$, and mean box size $b_{m}$.

$$
b_{\max }=\operatorname{round}(\min (H, W) / 2)
$$

where $\mathrm{H}$ and $\mathrm{W}$ represents number of rows and columns of the image respectively.

$$
b_{m}=\operatorname{round}\left(\left(b_{\min }+b_{\max }\right) / 2\right)
$$

Let us consider $b_{1}=b_{\min }, b_{2}=b_{m}$, and $b_{3}=b_{\max }$ for notational convenience. The number and size of boxes are decided empirically. Guided filter with smaller box size performs less smoothening and preserves more details, while larger box sizes provide more smoothening. In this approach, our motive is to obtain illumination details for images with spatially varying illumination. It is established by various researchers $[18,19,20]$ that maximum intensity across all color channels (i.e., bright-channel) provides a reasonable estimation of the illumination. However, it has some limitations like generation of unwanted artifacts, dispersion of light in light dominant areas, over enhancement in bright regions, etc. The proposed algorithm considers the maximum of all smooth versions (i.e., all box sizes) of the input image, to get a structure-aware 


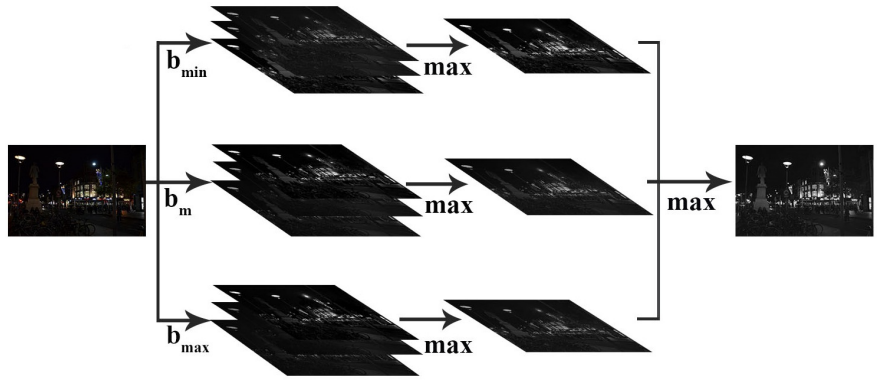

Fig. 2: Framework of Structure-Aware Initial Illumination Estimation. In first step, guided filters with box sizes $b_{\min }, b_{m}$, and $b_{\max }$ are applied to the low-light image. Then, the pixel-wise max operation is performed across all three color channels on each output images of guided filters. This results in three max channels corresponding to each box size (lets call them as max guided channels.). Finally, the pixel-wise max operation is applied on above three max guided channels to achieve the structure-aware initial illumination.

estimation of illumination. The proposed algorithm computes initial estimation as given below:

$$
\hat{\mathbf{I}}=\max _{i}\left\{\bigcup_{i=1}^{3} \max _{c \in\{R, G, B\}}\left\{\mathbf{J}_{b_{i}}^{c}\right\}\right\}
$$

where $\hat{\mathbf{I}}$ denotes the initial illumination, $\mathbf{J}_{b_{i}}^{c}$ denotes $c^{\text {th }}$ color channel of the output image of guided filter with box size $b_{i}$, where $\mathrm{i}=1,2,3$ and $\mathrm{R}, \mathrm{G}, \mathrm{B}$ denotes red, green \& blue component of the color image. The framework of structureaware initial illumination estimation are shown in Fig. 2.

Indeed, there are two extreme cases of illumination estimations: (i). smooth image by applying center/surround functions such as Gaussian filtering, and (ii). select the maximum of all three color channels of the input image. In the first extreme case, estimated illumination fails to capture spatial variations in actual illumination. While in the second case, initial estimation fails to limit the effect of dominating intensities such as artificial light sources, and thereby produces a scattering effect. Hence, in the proposed algorithm, we maintain the trade-off between these extremities. We consider a maximum of smooth versions of all color channels, which prevents the aforementioned biases and produces a better estimation of the actual illumination. The proposed initial illumination gives a reasonably good estimation of actual illumination and results in good enhancement. However, the estimated illumination still contains textural details and may result in over-enhanced images. Thus, there is a need for smoothening of the initial illumination while preserving the structural details. To achieve a structure-aware smoothening, we formulate the following multi-objective optimization problem:

$$
\min _{\mathbf{I}}\left\|\hat{\mathbf{I}}-\mathbf{I}||_{2}^{2}+\alpha\right\| \nabla \mathbf{J}_{m}-\nabla \mathbf{I}\left\|_{2}^{2}+\beta\right\| \mathbf{G} \circ \nabla \mathbf{I} \|_{1}
$$

where $\mathbf{I}$ is the refined illumination, $\hat{\mathbf{I}}$ is initial illumination as obtained by (4), $\nabla$ denotes first-order derivative operator, $\nabla \mathbf{J}_{m}$ denotes maximum of $\nabla$ from all channels of $\mathbf{J}$ (i.e., input image), $\mathbf{G}$ is a weight matrix based on initial illumination as discussed later in this section, $\|\bullet\|_{p}$ represents $L^{p}$ norm operator, and $\alpha \& \beta$ are the regularization parameters.

The first term (i.e., ||$\hat{\mathbf{I}}-\mathbf{I} \|_{2}^{2}$ ) of (5) provides fidelity between initial illumination and refined illumination. The second term (i.e., $\left\|\nabla \mathbf{J}_{m}-\nabla \mathbf{I}\right\|_{2}^{2}$ ) pulls the gradient of the refined illumination towards the maximum gradient of input images $\left(\nabla \mathbf{J}_{m}\right)$. It tries to maximize the structural details in the illumination. The third term (i.e., $\|\mathbf{G} \circ \nabla \mathbf{I}\|_{1}$ ) is used to provide smoothness to minimize textural details. The second term of (5) limits the smoothing effect introduced by the third term, while the third term prevents the second term from enhancing textural or noisy details. Thus, a combination of the second and third term results in preserving structural details, and smoothening textural details in the refined illumination.

In the third term, weight matrix $\mathbf{G}$ plays a vital role in smoothening of the textural details. The weights in matrix $\mathbf{G}$ are calculated as below:

$$
\begin{aligned}
& \mathbf{G}_{x}=-\log \left(\nabla_{x} \hat{\mathbf{I}}\right) \\
& \mathbf{G}_{y}=-\log \left(\nabla_{y} \hat{\mathbf{I}}\right)
\end{aligned}
$$

where $\nabla_{x}$ denotes first-order derivative along horizontal dimension and $\nabla_{y}$ denotes first-order derivative along vertical dimension. The logarithmic of derivative in both directions assign a higher weight to textural details, while reducing the importance of structural details. Note that low gradient values characterize texture details, while high gradient values characterize structural details. Thus, the minimization of the multiobjective function significantly suppresses textural details and result in textural smoothening, while reducing the smoothening effect in structural details.

\section{Solution of the optimization problem}

The efficient solution of the problem (5) can be obtained using the Alternative Direction Minimization (ADM) algorithm [22]. The researchers $[15,17,20]$ have proven the convergence of optimization problem like in (5) to the global optimum. Let us replace the term $\nabla \mathbf{I}$ with an auxiliary variable $\mathbf{K}$ and rewrite objective function in (5) as:

$$
\begin{array}{r}
\underset{\mathbf{I}, \mathbf{K}}{\arg \min }\|\hat{\mathbf{I}}-\mathbf{I}\|_{2}^{2}+\alpha\left\|\nabla \mathbf{J}_{m}-\mathbf{K}\right\|_{2}^{2}+\beta\|\mathbf{G} \circ \mathbf{K}\|_{1} \\
\text { s.t. } \nabla \mathbf{I}=\mathbf{K}
\end{array}
$$

The multi-objective optimization which contains equality constraint can use Lagrangian multiplier to compute the optimum point. The equality constraint in (8) can be included in the objective function using Lagrangian multiplier $(\mathbf{L})$. The augmented Lagrangian function of (8) is given as:

$$
\begin{array}{r}
\mathcal{L}=\|\hat{\mathbf{I}}-\mathbf{I}\|_{2}^{2}+\alpha\left\|\nabla \mathbf{J}_{m}-\mathbf{K}\right\|_{2}^{2}+\beta\|\mathbf{G} \circ \mathbf{K}\|_{1} \\
+\varphi(\mathbf{L}, \nabla \mathbf{I}-\mathbf{K})
\end{array}
$$

where $\varphi(\mathbf{L}, \nabla \mathbf{I}-\mathbf{K})=\frac{\omega}{2}\|\nabla \mathbf{I}-\mathbf{K}\|_{2}^{2}+\langle\mathbf{L}, \nabla \mathbf{I}-\mathbf{K}\rangle$ and $\langle\cdot, \cdot\rangle$ denotes element-wise multiplication, $\omega$ is a positive penalty term. The penalty term is required to control the rate of convergence to the solution [22]. The optimization problem can be solved easily by iteratively updating one variable while considering others as constant. The optimization problem (5) can be simplified by separating it into sub-problems. We divide the proposed multi-objective function into two sub-problems, I sub-problem, and $\mathbf{K}$ sub-problem. I sub-problem is the formed by considering the all terms of (9) which contain illumination 
component. $\mathbf{K}$ sub-problem is the formed by considering the all terms of (9) which contain equality constraint. Let us solve both sub-problems using ADM[22] algorithm.

I sub-problem: Considering only terms related to the variable I of (9), we obtain the following sub-problem at $i^{\text {th }}$ iteration:

$$
\mathbf{I}^{i+1}=\underset{I}{\arg \min }\|\hat{\mathbf{I}}-\mathbf{I}\|_{2}^{2}+\varphi\left(\mathbf{L}^{i}, \nabla \mathbf{I}-\mathbf{K}^{i}\right)
$$

This is a well-known least square problem and can be solved by differentiating it w.r.t. to I and putting it equal to 0 :

$$
\begin{gathered}
2(\hat{\mathbf{I}}-\mathbf{I})+\omega^{i} \mathbf{D}^{T}\left(\mathbf{D I}-\mathbf{K}^{i}\right)+\mathbf{D}^{T} \mathbf{L}^{i}=0 \\
\mathbf{I}=\frac{2 \hat{\mathbf{I}}+\mathbf{D}^{T}(\omega \mathbf{K}-\mathbf{L})}{\mathbf{2}+\omega \mathbf{D}^{T} \mathbf{D}}
\end{gathered}
$$

where D denotes the matrix containing Dx and Dy, Dx is the difference along rows and Dy is the difference along columns. Multiplication, transpose, and inverses of large matrices may be computationally costly. Thus, we use the 2D-FFT method by considering the condition of the circular boundary, and compute I as:

$$
\mathbf{I}^{i+1}=\mathcal{F}^{-1}\left(\frac{\mathcal{F}(2 \hat{\mathbf{I}})+\sum_{d \in\{x, y\}} \mathcal{F}^{c}\left(\mathbf{D}_{d}\left(\omega^{i} \mathbf{K}^{i}-\mathbf{L}^{i}\right)\right)}{\mathbf{2}+\omega^{i} \sum_{d \in\{x, y\}} \mathcal{F}^{c}\left(\mathbf{D}_{d}\right) \cdot \mathcal{F}\left(\mathbf{D}_{d}\right)}\right)
$$

where $\mathcal{F}$ denotes 2D-FFT operation, $\mathcal{F}^{c}$ denotes complex conjugate of $2 \mathrm{D}$-FFT operation, $\mathcal{F}^{-1}$ denotes the inverse of 2D-FFT operation and $\mathbf{2}$ is a matrix equivalent to image size having all elements 2. Here, all operations are element-wise. K sub-problem: K sub-problem is obtained from (9) by selecting terms related to $\mathbf{K}$ only. Thus, we have the following sub-problem at $i^{\text {th }}$ iteration:

$$
\begin{array}{r}
\mathbf{K}^{i+1}=\underset{K}{\arg \min } \alpha\left\|\nabla \mathbf{J}_{m}-\mathbf{K}\right\|_{2}^{2}+\beta\|\mathbf{G} \circ \mathbf{K}\|_{1} \\
+\frac{\omega}{2}\|\nabla \mathbf{I}-\mathbf{K}\|_{2}^{2}+\langle\mathbf{L}, \nabla \mathbf{I}-\mathbf{K}\rangle
\end{array}
$$

To solve the minimization problem (14) take derivative with respect to $\mathbf{K}$ and equate it to zero.

$$
\begin{gathered}
\mathbf{K}(2 \alpha+\omega)-2 \alpha \nabla \mathbf{J}_{m}+\beta \mathbf{G}-\omega \nabla \mathbf{I}-\mathbf{L}=0 \\
\mathbf{K}=\frac{2 \alpha \nabla \mathbf{J}_{m}+\omega \nabla \mathbf{I}+\mathbf{L}}{(2 \alpha+\omega)}-\frac{\beta \mathbf{G}}{(2 \alpha+\omega)}
\end{gathered}
$$

The value of a pixel in an image cannot be negative. However, due to higher weights, $\mathbf{K}$ can have negative pixel values if computation uses direct subtraction of weights as (16), which is inappropriate while dealing with images. Thus, the computation of $\mathbf{K}$ requires normalization to deal with negative pixel values. Shrinkage operation like in $[17,20]$ helps in thresholding the values of the image. Hence, we can solve $\mathrm{K}$ sub-problem with the help of shrinkage operation:

$$
\mathbf{K}^{i+1}=\mathcal{S}_{\frac{\beta \cdot \mathbf{G}}{2 \alpha+\omega^{i}}}\left[\frac{2 \alpha \nabla \mathbf{J}_{m}+\omega \nabla \mathbf{I}^{i+1}+\mathbf{L}^{i}}{2 \alpha+\omega^{i}}\right]
$$

where $\mathcal{S}_{\phi}[x]=\operatorname{sign}(x) \max (|x|-\phi, 0)$ and, all operations are element-wise. The values of parameters $\alpha$ and $\beta$ are chosen empirically. The parameters analysis shows that the best values of $\alpha$ and $\beta$ are 0.5 , and 0.1 respectively. The detailed analysis is discussed in section III-C.

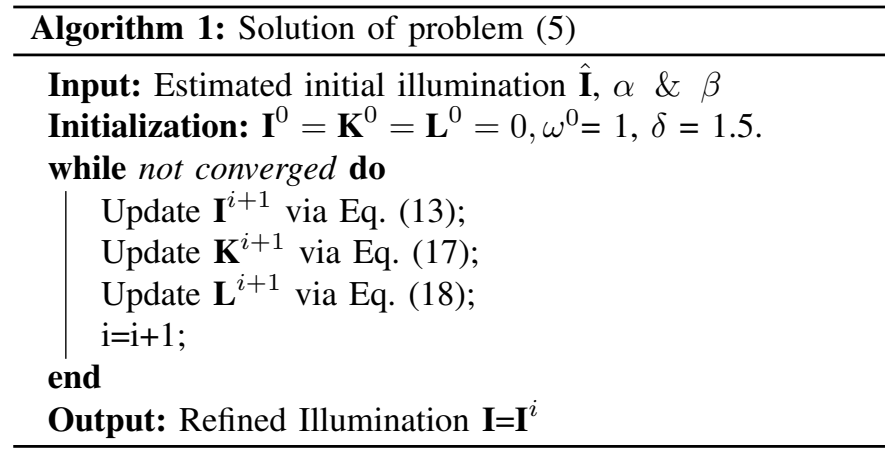

$\mathbf{L}$ and $\omega$ : The Lagrangian multiplier matrix $\mathbf{L}$ and penalty term $\omega$ can be updated as follows:

$$
\begin{aligned}
& \mathbf{L}^{i+1}=\mathbf{L}^{i}+\omega^{i}\left(\nabla \mathbf{I}^{i+1}-\mathbf{K}^{i+1}\right) ; \\
& \omega^{i+1}=\omega^{i} \delta, \delta>1 .
\end{aligned}
$$

The above iterative algorithm needs suitable stopping criteria to achieve an optimal solution. We can either consider a threshold for the difference between $\mathbf{I}^{i+1}$ and $\mathbf{I}^{i}$ (i.e., $I_{\text {error }}^{i}$ ) or work out the maximum number of iteration. We have considered the second option and find the maximum number of iteration using a large number of experiments with various image datasets. We analyze the results and found that after eight iterations, the proposed algorithm gives optimal results, as shown in Fig. 11. If we consider threshold difference many times algorithm runs a larger number of iteration without significant improvement, and results in the wastage of computation power. Moreover, finding a fixed (optimal) number of iteration helps in keeping time complexity independent of the number of iteration. The whole procedure of estimating refined illumination is summarized in Algorithm 1, which covers all details regarding variables initialization.

After estimation of illumination component for input image, we require to find reflectance components. Remember illumination component is independent of color channel, however reflectance component will depend on color channel. The estimated reflectance components are given as:

$$
\mathbf{R}^{c}=\mathbf{J}^{c} \cdot / \mathbf{I}
$$

where $\mathbf{R}^{c}$ is estimated reflectance of $c^{t h}$ color channel and division is element-wise.

To further improve the visibility of the input image, the range illumination is adjusted using gamma correction. The final enhanced image $\mathbf{J}_{f}^{c}$ is given as:

$$
\mathbf{J}_{f}^{c}=\mathbf{R}^{c} \circ \mathbf{I}_{f}
$$

where $\mathbf{I}_{f}$ is gamma-corrected illumination estimation.

\section{EXPERIMENTAL RESULTS AND ANALYSIS}

The proposed algorithm is analyzed and validated experimentally. The performance is evaluated with both Quantitative and Visual assessments. The time complexity is another important aspect to assess the performance of an algorithm. Thus, the computational cost is also analyzed for the proposed approach. We tested the proposed method using more than 200 

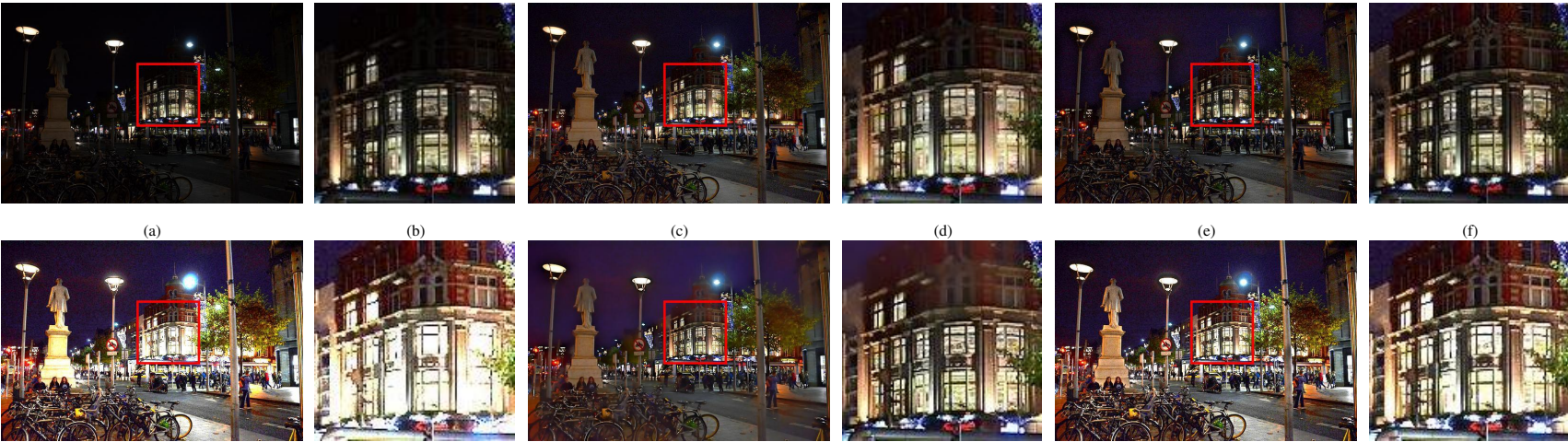

(h)
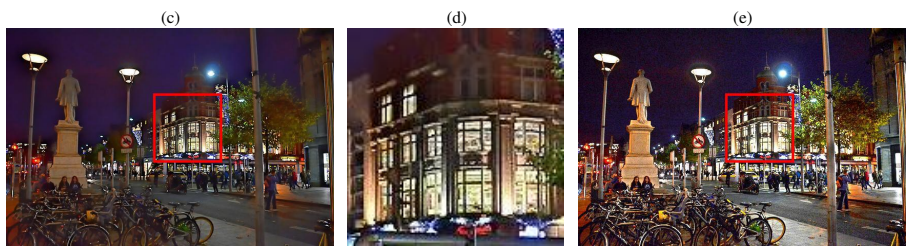

(k)

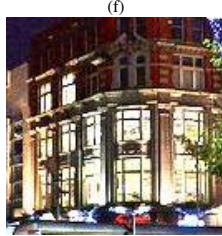

Fig. 3: Street. (a) Input image; (b) Marked patch of (a); (c) Result of PIRE; (d) marked patch of (c); (e) Result of WRIE; (f) Marked patch of (e); (g) Result of LIME; (h) Marked patch of (g); (i) Result of SRLIME; (j) Marked patch of (i); (k) Result of proposed method; (l) Marked patch of (k).

images (having varying illumination) taken from various image datasets: ExDark Dataset [23], HDR Dataset [24], Kodak Dataset [25], NASA Database [26] and Berkeley Segmentation Dataset [27]. We compare the performance of the proposed algorithm with other state-of-the-art algorithms: PIRE [15], WRIE [16], LIME [20] and SRLIME [17]. We have used codes and parameters (of other algorithms) as available on the author's websites/or as provided by authors for a fair comparison.

\section{A. Quantitative Assessment}

The quantitative assessment is always desirable to validate any experimental result. However, it is a challenging task to develop a quantitative measure in case of image enhancement. There have been several attempts to design such a measure. It may be easily verified that a quantitative measure may give better value for poorly enhanced image and inferior value for a high quality enhanced image and vice-a-versa. There is no single universally accepted quantitative assessment method available. Thus, we have used two quantitative methods (mostly used in other works): Contrast Gain (CG) [15], and Structure-aware Lightness Order Error (SLOE).

Contrast Gain [15] is the ratio of the contrast of an enhanced image to an original image.

$$
C G=\frac{C_{\text {enhanced }}}{C_{\text {input }}}
$$

where $C_{\text {enhanced }}$ denotes weber contrast of an enhanced image and $C_{\text {input }}$ denotes weber contrast of input image.

TABLE I: CONTRAST GAIN VALUES FOR QUANTITATIVE MEASURE

\begin{tabular}{lllllll}
\hline Image & MSR & PIRE & WRIE & LIME & SRLIME & Proposed \\
\hline City & 0.33 & 0.75 & 0.79 & 0.78 & 0.57 & $\mathbf{0 . 8 4}$ \\
Landscape & 0.61 & 0.85 & 0.95 & 0.92 & 0.55 & $\mathbf{0 . 9 8}$ \\
Street & 0.43 & 0.88 & 0.97 & 0.88 & 0.56 & $\mathbf{0 . 9 9}$ \\
Rider & 0.52 & 0.93 & 1.00 & 0.97 & 0.33 & $\mathbf{1 . 0 4}$ \\
Dog & 0.45 & 0.83 & 0.97 & 0.94 & 0.53 & $\mathbf{0 . 9 9}$ \\
Swing & 0.50 & 0.79 & 0.85 & 0.83 & 0.75 & $\mathbf{0 . 8 6}$ \\
Girl & 0.36 & 0.78 & 0.84 & 0.79 & 0.58 & $\mathbf{0 . 8 8}$ \\
Window & 0.40 & 0.81 & 0.90 & 0.85 & 0.59 & $\mathbf{0 . 9 3}$ \\
House & 0.36 & 0.87 & $\mathbf{0 . 9 3}$ & 0.88 & 0.64 & 0.92 \\
Taxi & 0.43 & 0.86 & 0.91 & 0.89 & 0.71 & $\mathbf{0 . 9 2}$ \\
\hline Mean & 0.44 & 0.84 & 0.91 & 0.87 & 0.58 & $\mathbf{0 . 9 4}$ \\
\hline
\end{tabular}

In general, a properly illuminated image should give a higher contrast value. Moreover, higher structural details contribute to better contrast. Thus, higher $\mathrm{CG}$ is desirable for enhanced images. The CG values for ten sample images out of more than 200 test images are shown in Table-I. The highest $\mathrm{CG}$ values are shown in bold for better reference. It may be noticed that the proposed algorithm gives the best $\mathrm{CG}$ values in most cases. The means of $\mathrm{CG}$ values of all images for various algorithms are shown at the bottom of the Table-I. The proposed algorithm gives the best mean CG value. It validates that the proposed algorithm gives better illumination and preserves more structural details in comparison to other algorithms.

However, the higher $\mathrm{CG}$ values sometimes may be due to enhanced noise or over enhancement of some regions of the image. Thus, we may use another quantitative measure, namely Lightness Order Error (LOE) [18]. LOE is an average of the relative order of lightness of pixels in input and enhanced images. LOE is given as:

$$
\begin{gathered}
L O E=\frac{1}{H * W} \sum_{x=1}^{H} \sum_{y=1}^{W} R O(x, y) \\
R O(x, y)=\sum_{u=1}^{H} \sum_{v=1}^{W} U(\bar{I}(x, y), \bar{I}(u, v)) \oplus U\left(\bar{I}_{f}(x, y), \bar{I}_{f}(u, v)\right) \\
U(a, b)= \begin{cases}1, & \text { if } a \geq b \\
0, & \text { otherwise }\end{cases} \\
\bar{I}(x, y)=\max _{c \in R, G, B}\left(J^{c}(x, y)\right)
\end{gathered}
$$

where $\oplus$ represents XOR operation, $\bar{I}$ and $\bar{I}_{f}$ is the lightness of input image and final enhanced image respectively. LOE is a measure of the number of pixels that maintained relative intensity order in the enhanced image. Thus, the lower LOE represents better image enhancment. LOE may be a good measure of enhancement for images with uniform illumination. However, the order of relative lightness may not be maintained in case of non-uniform illumination. It happens because some pixels in dark regions require a higher increase in the illumination, while pixels in properly illuminated areas may remain the same. For example, consider the images shown 

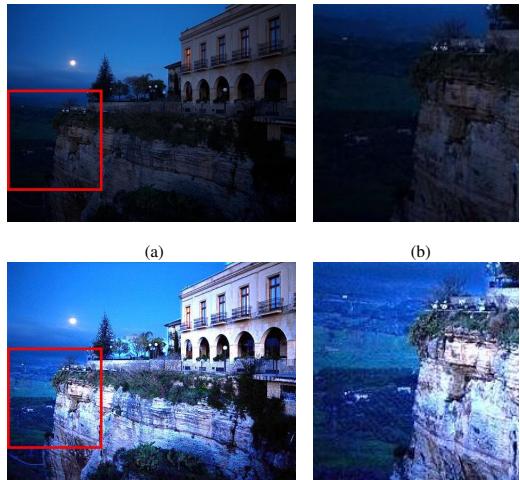

(g)

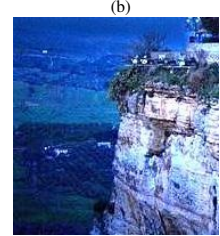

(h)
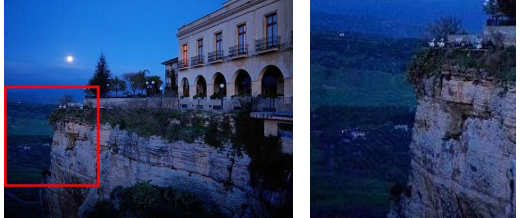

(d)

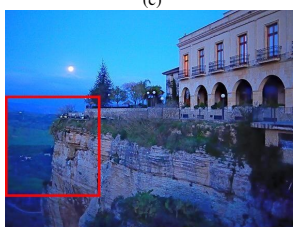

(i)

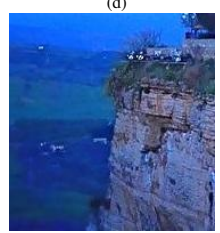

(j)

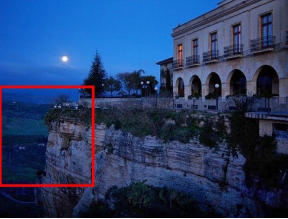

(e)

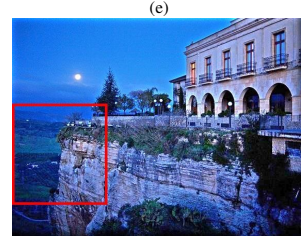

(k)

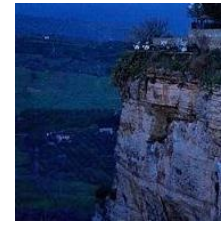

(f)

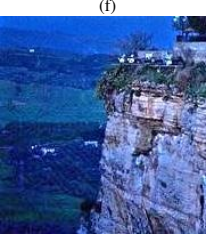

(1)

Fig. 4: Landscape. (a) Input image; (b) Marked patch of (a); (c) Result of PIRE; (d) marked patch of (c); (e) Result of WRIE; (f) Marked patch of (e); (g) Result of LIME; (h) Marked patch of (g); (i) Result of SRLIME; (j) Marked patch of (i); (k) Result of proposed method; (l) Marked patch of (k).
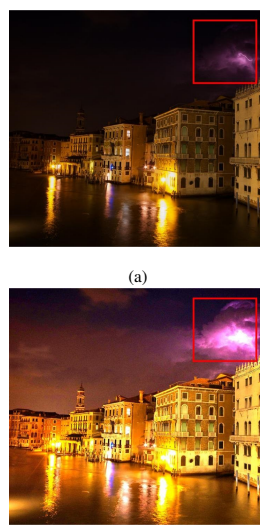

(g)
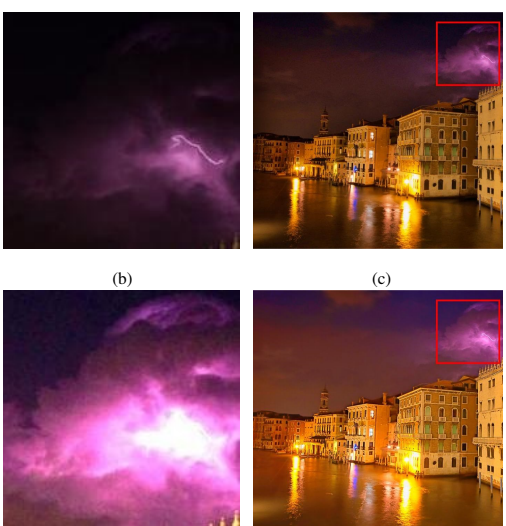

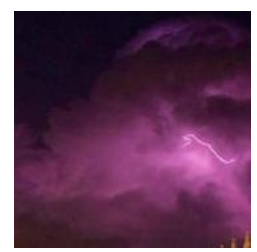

(d)

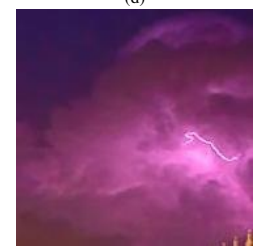

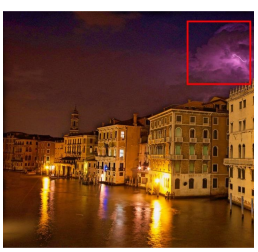

(e)
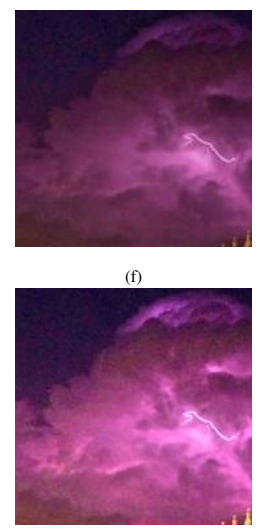

Fig. 5: City. (a) Input image; (b) Marked patch of (a); (c) Result of PIRE; (d) marked patch of (c); (e) Result of WRIE; (f) Marked patch of (e); (g) Result of LIME; (h) Marked patch of (g); (i) Result of SRLIME; (j) Marked patch of (i); (k) Result of proposed method; (l) Marked patch of (k).

in Fig. 6 and Fig. 7. It can be noted that LIME obtains the best LOE values (3.38, and 3.23, respectively), while the proposed algorithm gives LOE values: 4.17 and 12.11 for Fig. 6 and Fig. 7 respectively. However, it may be noted from both images that LIME gives over enhancement with some prominent artifacts. Thus, there is a poor correlation between the quality of enhanced image and LOE value. We proposed a modification to LOE by incorporating structural information of the image along with relative order of lightness. We consider structure-aware lightness $(\hat{I})$ instead of simple lightness $(\bar{I})$. Structure-aware lightness is given as:

$$
\hat{I}(x, y)=\max _{i}\left\{\bigcup_{i=1}^{3} \max _{c \in\{R, G, B\}}\left\{J_{b_{i}}^{c}(x, y)\right\}\right\}
$$

Indeed, the lightness defined in (25) is the same as the initial illumination estimation used in various algorithms $[18,19,20]$. The SLOE uses structure-aware lightness (defined in (4) and (26)), thus it have the same advantages over simple lightness as discussed in section II-A. We consider images from NASA [26] and LOL [28] database as these have reference images with good illumination. The SLOE values for various methods are shown in Table-II. It may be noticed that the proposed method gives best SLOE values for the majority of the images with few exceptions. It is found that there is much better correlation between visual quality of enhanced image and its
SLOE values than LOE values. In Table-II, WRIE [16] gives better SLOE value than the proposed algorithm. However, it may be noted from Fig.6 (c) and (j) that the proposed algorithm gives much better and natural enhancement than WRIE.

\section{B. Visual Assessment}

As discussed in the earlier section that there is no universally accepted quantitative measure for the assessment of the enhancement. Almost every method fails in one or other situations. Thus, a visual assessment of the results becomes a necessity. An exhaustive visual assessment of the results

TABLE II: SLOE VALUES FOR QUANTITATIVE MEASURE

\begin{tabular}{lllllll}
\hline Image & MSR & PIRE & WRIE & LIME & SRLIME & Proposed \\
\hline Book & 3.40 & 3.01 & 3.24 & 4.25 & $\mathbf{2 . 5 1}$ & 3.40 \\
Closet & 5.92 & $\mathbf{3 . 5 3}$ & 3.57 & 3.53 & 4.83 & 5.78 \\
Machine & 4.94 & 4.37 & 4.66 & 4.64 & 4.67 & $\mathbf{4 . 2 5}$ \\
Pool & 2.59 & 2.33 & 2.59 & 3.54 & 2.34 & $\mathbf{2 . 3 3}$ \\
Stands & 3.17 & 2.76 & 2.99 & 4.30 & 3.24 & $\mathbf{2 . 7 5}$ \\
Swing & 4.23 & 3.46 & 2.90 & 5.08 & 3.84 & $\mathbf{2 . 8 6}$ \\
Dress & 3.95 & 3.75 & 3.83 & 4.78 & 3.34 & $\mathbf{2 . 6 6}$ \\
Girl & 7.73 & 4.95 & $\mathbf{4 . 3 2}$ & 8.13 & 9.38 & 6.41 \\
House & 7.32 & 7.43 & 6.89 & 11.47 & 6.25 & $\mathbf{5 . 1 9}$ \\
Shoe & 5.02 & 4.62 & 4.42 & 5.79 & 3.52 & $\mathbf{3 . 2 4}$ \\
\hline Mean & 4.83 & 4.02 & 3.94 & 5.55 & 4.39 & $\mathbf{3 . 5 9}$ \\
\hline
\end{tabular}



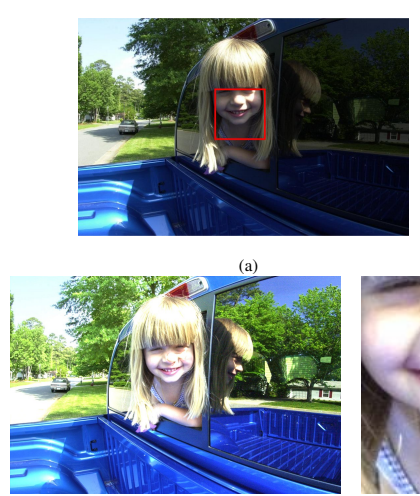

(f)

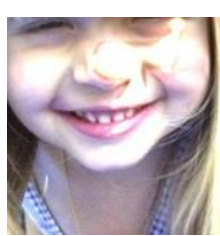

$(\mathrm{g})$
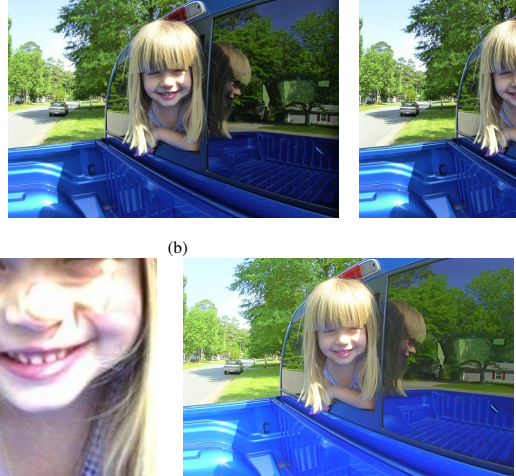

(h)
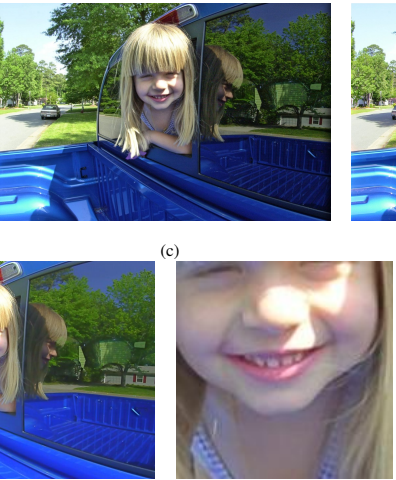

(i)
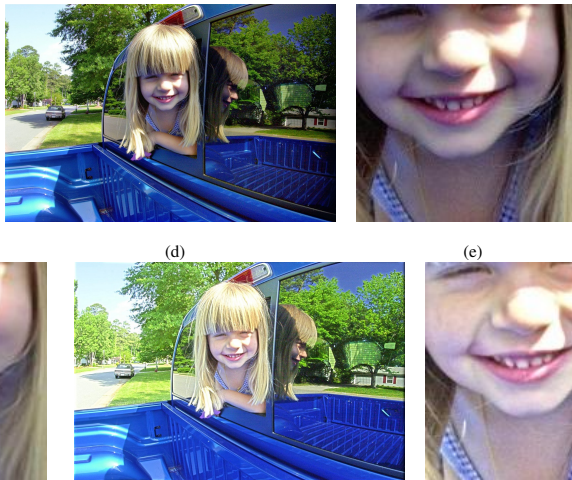

(j)

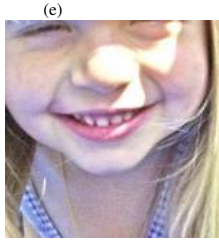

(k)

Fig. 6: Girl. (a) Input image; (b) Result of PIRE; (c) Result of WRIE; (d) Result of NASA; (e) patch of (d); (f) Result of LIME; (g) patch of (f); (h) Result of SRLIME; (i) patch of (h); (j) Result of proposed method, (k) patch of (j).
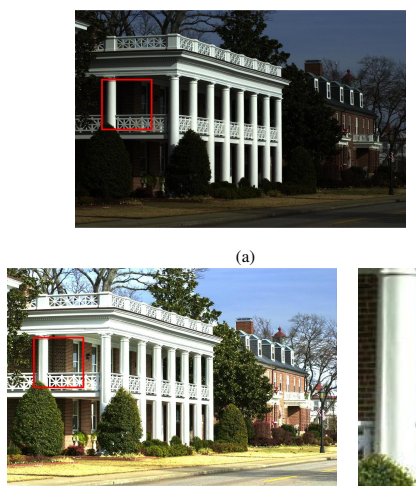

(f)
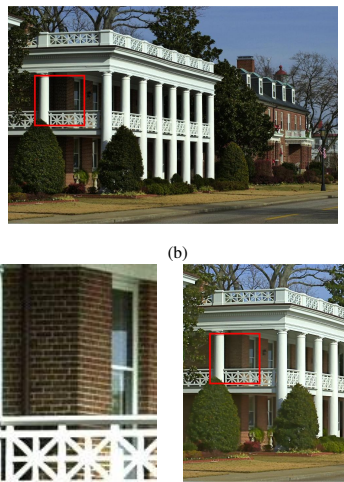

(g)
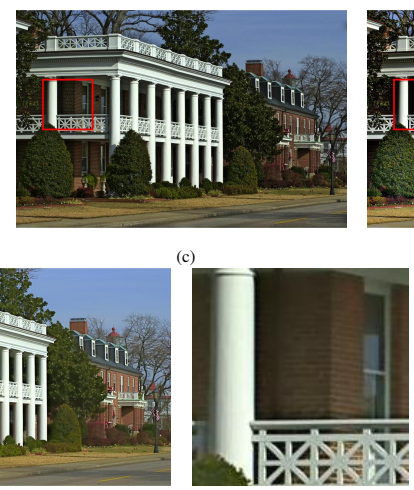

(i)
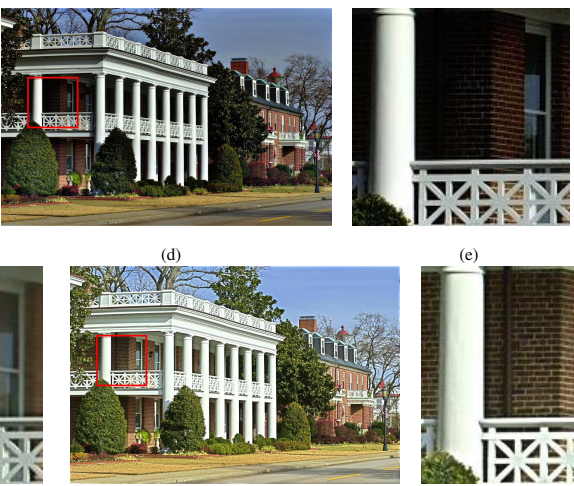

(j)

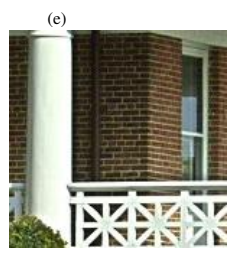

(k)

Fig. 7: House. (a) Input image; (b) Result of PIRE; (c) Result of WRIE; (d) Result of NASA; (e) patch of (d); (f) Result of LIME; (g) patch of (f); (h) Result of SRLIME; (i) patch of (h); (j) Result of proposed method, (k) patch of (j).

obtained by the proposed algorithm and other contemporary algorithms is performed. The sample results are shown in Fig. 3-8.

Fig. 3 shows a dark image 'street' and enhanced images by various algorithms. It may be observed from Fig. 3 (c)-(f), that algorithms PIRE and WRIE are unable to give significant enhancement. Image details are not visible in many regions of the images for example highlighted regions in red boxes. Fig. 3(g) shows that LIME gives relatively better enhancement. However, light dominated regions get over enhanced as shown in Fig. 3(h). It may be noticed that details are hardly visible in the window region. LIME gives over enhancement as shown in Fig. 3(g). It fails, especially in light dominant regions. As highlighted in Fig. 3(h), details in the window region are hardly visible. Fig. 3(i) shows an enhanced image by SRLIME algorithm. SRLIME focuses on noise removal that may be present in dark images. However, it smooths out natural details as well and produces a blurring effect. One may notice blurred details in the cycle stand in Fig. 3(i) and upper red color region in Fig. 3(j). The proposed algorithm provides good enhancement with almost no artifacts. Fig. 3(k) and 3(1) show that the scattering of light is restricted and details are preserved. In other words, low light regions are lightened up properly, while already lightened regions are not over enhanced as in the case of LIME. Moreover, Fig. 3(k) shows that the proposed algorithm effectively preserves color constancy.

A low-light image 'Landscape' and its enhanced versions by various algorithms are shown in Fig. 4. It may be noticed that PIRE and WRIE produce natural quality images, but lightness is not much improved. Fig. 4(g) and 4(h) show results by LIME algorithm. It shows good enhancement of details, but over enhancement may be noticed in patches as shown in Fig. 4(h). SRLIME gives images with improved visibility. However, the blurring effect and fading of colors may be seen in Fig. 4(i) and 4(j). The results of the proposed algorithm are shown in Fig. 4(k) and 4(1). It can be noticed that image details are much more clear with color constancy.

Fig. 5 shows a low-light image 'City' with non-uniform illumination and the results of various algorithms. The red box highlights thunder lightning in clouds. PIRE and WRIE are unable to enhance low-light regions significantly. LIME again provides reasonably good enhancement in dark regions of the image. However, it produces dispersion effect in thunder lightening region as highlighted in red box, see Fig. 5(g) and 5(h). The artifact is highlighted in Fig. 5(d), where thunder lightning is dispersed in large cloud region. Fig. 5(i) and 5(j) show the results of SRLIME algorithm. It gives satisfactory enhancement, but produce smoothening of the image details. It may be noted in Fig. 5(i) that cloud texture details are wiped out. The proposed algorithm gives significant enhancement while preserving natural texture details. 


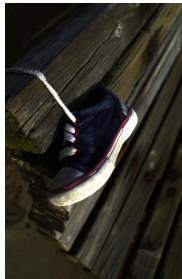

(a)

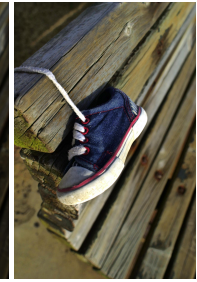

(b)

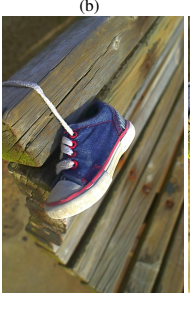

(e)

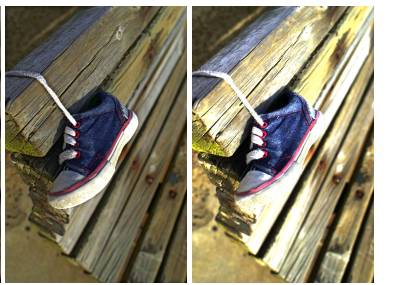

(d)

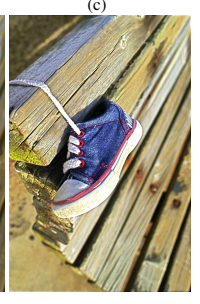

Fig. 8: Shoe. (a) Original Image; (b) Result of PIRE; (c) Result of WRIE; (d) Result of LIME, (e) Result of SRLIME; (f) Result of Proposed method.

An image 'Girl' with varying illumination and its enhanced versions by the various algorithm are shown in Fig. 6. Original image has a shaded region with low visibility, and other areas have typical daylight visibility. PIRE and WRIE provide limited visibility improvement in the shaded areas. It may be noted from Fig. 6(b) and 6(c) that reflection in car glass has low visibility. NASA results are shown in Fig. 6(j) and (k). It shows significantly improved visibility without any artifacts. LIME gives much-improved visibility in the shaded region as well. However, it may be noted from Fig. 6(g) that LIME introduces artifacts, especially near the nose of the girl. SRLIME also gives improved visibility, but Fig. 6(h) and 6(i) show that face texture and color of images are disturbed. The proposed algorithm gives much-improved visibility in all regions without any artifacts. It may be observed from Fig. 6(j) and $(\mathrm{k})$ that color constancy of the image is also preserved.

Fig. 7 shows a low-light image 'House' with varying illumination, and results of various enhancement algorithms. One can notice that PIRE, WRIE, and NASA image have almost similar enhancement with NASA image having slightly better contrast. Fig. 7(a-e) shows that these algorithms fail to improve visibility in the shaded regions. The result of LIME in Fig. 7(f), shows that it gives much-improved visibility. However, artifacts are introduced in the enhanced image as indicated in Fig. 7(g). SRLIME again gives improved visibility but vanishes texture as well as some structural details as shown in Fig. 7(h) and 7(i). The proposed algorithm gives improved visibility even in a shaded region as shown in Fig. 7(j) and $7(\mathrm{k})$. It may be noticed that the proposed algorithm provides enhancement without disturbing natural colors.

In Fig. 8 another shaded image 'shoe' and its enhanced results are shown. We again find similar kinds of effects as in other cases. PIRE and WRIE give less improvement visibility. LIME gives much-improved visibility, but over enhancement may be noticed as near the sole of the shoe in Fig. 8(d). SRLIME produces the washout effect due to the loss of the textural details. Fig. 8(f) shows that the proposed algorithm again gives much-improved visibility without any artifacts.

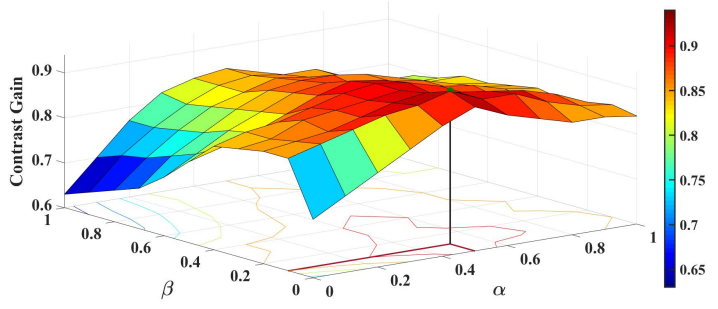

Fig. 9: Mean contrast gain for different pairs of $(\alpha, \beta)$. The contour denotes that pairs inside the contour have values based on the contour color. The colorbar represents the value of the contrast gain with respect to the color from the surface plot.

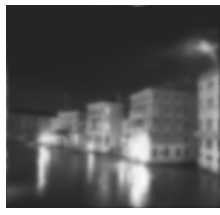

(a)

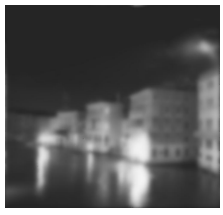

(b)

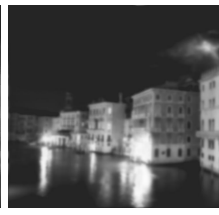

(c)

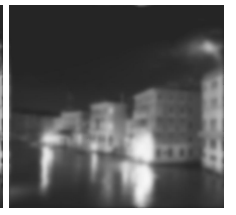

(d)
Fig. 10: Effects of Regularization parameters on illumination Estimation. (a) $\alpha=0.5$, $\beta=0.1$; (b) $\alpha=0.5, \beta=1$; (c) $\alpha=1, \beta=0.1$; (d) $\alpha=1, \beta=1$.

\section{Parameter Analysis}

The proposed algorithm involves two regularization parameters $\alpha$ and $\beta$. To analyze the effect of these parameters, we performed exhaustive experimentation and it is found that the values of $\alpha$ and $\beta$ should vary between 0 and 1. Since the optimization problem (5) is multi-objective optimization, considering negative values of $\alpha$ and $\beta$ does not make any sense. As discussed in section II-B, parameters $\alpha$ and $\beta$ regularize structural information and smoothening of textural details respectively. Thus, if either of the parameter $\alpha$ and $\beta$ have value greater than 1 then one of the objective (textural or structural details) will be dominant. For example, if parameter $\alpha$ is increased beyond value 1 , the structural details will be dominant. Moreover, while capturing structural details, textural details may also get enhanced due to use of the gradients in the related term. It may result in unnatural enhanced images. Similarly, large value of $\beta$ may result in smoothening of structural details as well. This results in oversmoothen refined illumination. To avoid the dominance of the term related to regularization parameter and limit the adverse effect we consider values of $\alpha$ and $\beta$ between 0 and 1. To arrive at optimal values of the parameters $\alpha$ and $\beta$, we perform experimentation over 50 images of diverse illumination and consider their mean contrast gain as measure of enhancement. However, to have better correlation between image qualities and values, we also analyze best values using visual analysis. The results are plotted in Fig. 9. It may be noted that the best values of $\alpha$ and $\beta$ are 0.5 and 0.1 respectively. Thus at $\alpha=0.5$, better structural details are captured without overenhancing textural details. In case of $\alpha$ values less than 0.5 , structural information of the image is not captured adequately. On the other hand, for $\alpha$ greater than 0.5 , textural details are captured along with structural details. The parameter $\beta$ at value 0.1 provides desirable smoothening of textural details without losing structural details. For case of $\beta$ less than 0.1 , 


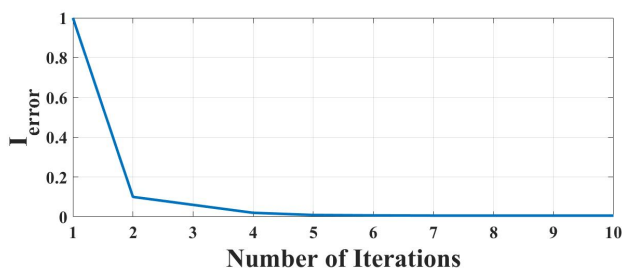

Fig. 11: Convergence curve for proposed Algorithm.

TABLE III: AVERAGE COMPUTATIONAL TIME PER IMAGE (IN SECONDS)

\begin{tabular}{lllll}
\hline PIRE & WRIE & LIME & SRLIME & Proposed \\
\hline 0.22 & 2.23 & 0.27 & 4.27 & 0.28 \\
\hline
\end{tabular}

smoothening of textural details is inadequate, while in case of $\beta$ greater than 0.1 smoothening of structural details occurs as well. Fig. 10 shows the estimated illumination for few distinct values of $\alpha=0.5$, and $\beta=0.1$. In our experimentation, the empirical values of parameters generate satisfactory results.

\section{Computational Analysis}

The computational time complexity plays an important role in the performance analysis of any algorithm. Thus, the Computational time analysis of the various algorithm is performed on a set of 200 images with size 640x424. Table III shows the average computational time per image. We use MATLAB 2018a on Windows10 running at 8GB RAM and core i5 processor @ 3.40GHz. WRIE and SRLIME are slower than other algorithms. These algorithms are not useful for Real-Time applications due to slow convergence. PIRE and LIME take slightly lesser time than the proposed algorithm. However, the visual quality of enhanced images produced by the proposed method are much better than PIRE and LIME. Further, let us analyze the time complexities of various algorithms for an image with $\mathrm{N}$ number of pixels. In case of PIRE, one iteration of estimation (both components: illumination \& reflectance) requires $\mathcal{O}(N)$ time, and its convergence requires $\mathcal{O}(\log N)$ iterations. Hence, total time complexity of PIRE is $\mathcal{O}(N \log N)$. In case of WRIE, one iteration of estimation (both components: illumination \& reflectance) requires $\mathcal{O}(N)$ time, and its convergence requires $\mathcal{O}(N)$ iterations. Hence, total time complexity of WRIE is $\mathcal{O}\left(N^{2}\right)$. In case of LIME, one iteration of estimation of illumination using Exact Solver requires $\mathcal{O}(N)$ time, and its convergence requires $\mathcal{O}(\log N)$ iterations. Hence, total time complexity of LIME is $\mathcal{O}(N \log N)$. In case of SRLIME, one iteration of Estimation (all components: illumination, reflectance and noise) requires $\mathcal{O}(N)$ time, and its convergence requires $\mathcal{O}(N)$ iterations. Hence, total time complexity of SRLIME is $\mathcal{O}\left(N^{2}\right)$. In case of proposed algorithm, one iteration of estimation of illumination requires $\mathcal{O}(N)$ time, and its convergence requires $\mathcal{O}(\log N)$ time. It requires comparable time as LIME.

\section{CONCLUSION}

In this paper, we presented a new approach for the estimation of illumination and reflectance from a low light image. The algorithm gives natural contrast enhancement for images with varying lighting. The experimental analysis shows that the proposed algorithm improves darks regions of the image without over-enhancing properly illuminated areas of the same image. Our algorithm has various advantages over other methods, which are:

- Works effectively for images that contain both low-light and properly illuminated images with shaded regions.

- Produces nature preserving enhanced image with almost no artifacts.

- Restrict light dispersion in the light dominant region while improving luminance in the dark regions.

We performed exhaustive experimentation over a large number of images with different variations in the illumination conditions to check the performance of the proposed method. The visual assessment shows that the proposed method maintains color constancy over the object under varying light conditions. The proposed algorithm outperforms most of the state-of-theart low-light image enhancement algorithms.

\section{REFERENCES}

[1] R. C. Gonzalez and R. E. Woods, Digital Image Processing (3rd Edition). USA: Prentice-Hall, Inc., 2006.

[2] M. Abdullah-Al-Wadud, M. H. Kabir, M. A. Akber Dewan, and O. Chae, "A dynamic histogram equalization for image contrast enhancement," IEEE Transactions on Consumer Electronics, vol. 53, pp. 593-600, May 2007.

[3] A. S. Parihar and O. P. Verma, "Contrast enhancement using entropy-based dynamic sub-histogram equalisation," IET Image Processing, vol. 10, no. 11, pp. 799-808, 2016.

[4] T. Celik and T. Tjahjadi, "Contextual and variational contrast enhancement," IEEE Transactions on Image Processing, vol. 20, pp. 3431-3441, Dec 2011.

[5] T. Celik, "Spatial entropy-based global and local image contrast enhancement," IEEE Transactions on Image Processing, vol. 23, pp. 5298-5308, Dec 2014.

[6] C. Lee, C. Lee, and C. Kim, "Contrast enhancement based on layered difference representation of $2 \mathrm{~d}$ histograms," IEEE Transactions on Image Processing, vol. 22, pp. 5372-5384, Dec 2013.

[7] A. S. Parihar, O. P. Verma, and C. Khanna, "Fuzzycontextual contrast enhancement," IEEE Transactions on Image Processing, vol. 26, pp. 1810-1819, April 2017.

[8] E. H. Land and J. J. McCann, "Lightness and retinex theory," Journal of Optical Society of America, vol. 61, pp. 1-11, Jan 1971.

[9] E. H. Land, "Recent advances in retinex theory and some implications for cortical computations: color vision and the natural image.," Proceedings of the National Academy of Sciences of the United States of America, vol. 80, no. 16, p. 5163, 1983.

[10] E. H. Land, "Recent advances in retinex theory," in Central and peripheral mechanisms of colour vision, pp. 5-17, Springer, 1985.

[11] A. S. Parihar and K. Singh, "A study on retinex based method for image enhancement," in 2018 2nd International Conference on Inventive Systems and Control (ICISC), pp. 619-624, Jan 2018. 
[12] D. J. Jobson, Z. Rahman, and G. A. Woodell, "Properties and performance of a center/surround retinex," IEEE Transactions on Image Processing, vol. 6, pp. 451-462, March 1997.

[13] Z.-u. Rahman, D. J. Jobson, and G. A. Woodell, "Multiscale retinex for color image enhancement," in Proceedings of 3rd IEEE International Conference on Image Processing, vol. 3, pp. 1003-1006, IEEE, 1996.

[14] D. J. Jobson, Z. Rahman, and G. A. Woodell, "A multiscale retinex for bridging the gap between color images and the human observation of scenes," IEEE Transactions on Image Processing, vol. 6, pp. 965-976, July 1997.

[15] X. Fu, Y. Liao, D. Zeng, Y. Huang, X. Zhang, and $X$. Ding, "A probabilistic method for image enhancement with simultaneous illumination and reflectance estimation," IEEE Transactions on Image Processing, vol. 24, pp. 4965-4977, Dec 2015.

[16] X. Fu, D. Zeng, Y. Huang, X. Zhang, and X. Ding, "A weighted variational model for simultaneous reflectance and illumination estimation," in 2016 IEEE Conference on Computer Vision and Pattern Recognition (CVPR), pp. 2782-2790, June 2016.

[17] M. Li, J. Liu, W. Yang, X. Sun, and Z. Guo, "Structurerevealing low-light image enhancement via robust retinex model," IEEE Transactions on Image Processing, vol. 27, pp. 2828-2841, June 2018.

[18] S. Wang, J. Zheng, H. Hu, and B. Li, "Naturalness preserved enhancement algorithm for non-uniform illumination images," IEEE Transactions on Image Processing, vol. 22, pp. 3538-3548, Sep. 2013.

[19] X. Fu, D. Zeng, Y. Huang, Y. Liao, X. Ding, and J. Paisley, "A fusion-based enhancing method for weakly illuminated images," Signal Processing, vol. 129, pp. 8296, 2016.

[20] X. Guo, Y. Li, and H. Ling, "Lime: Low-light image enhancement via illumination map estimation," IEEE Transactions on Image Processing, vol. 26, pp. 982-993, Feb 2017.

[21] K. He, J. Sun, and X. Tang, "Guided image filtering," IEEE Transactions on Pattern Analysis and Machine Intelligence, vol. 35, pp. 1397-1409, June 2013.

[22] Y. Wang, W. Yin, and J. Zeng, "Global convergence of admm in nonconvex nonsmooth optimization," Journal of Scientific Computing, vol. 78, no. 1, pp. 29-63, 2019.

[23] Y. P. Loh and C. S. Chan, "Getting to know low-light images with the exclusively dark dataset," Computer Vision and Image Understanding, vol. 178, pp. $30-42$, 2019.

[24] S. Hasinoff, D. Sharlet, R. Geiss, A. Adams, J. T. Barron, F. Kainz, J. Chen, and M. Levoy, "Burst photography for high dynamic range and low-light imaging on mobile cameras," SIGGRAPH Asia, 2016.

[25] R. W. Franzen, "Kodak lossless true color image suite." http://r0k.us/graphics/kodak/. Accessed: 2019-08-13.

[26] G. Woodell, "Retinex image processing." https://dragon.larc.nasa.gov/retinex/pao/news/. Accessed: 2019-08-13.

[27] D. Martin, C. Fowlkes, D. Tal, J. Malik, et al., "A database of human segmented natural images and its application to evaluating segmentation algorithms and measuring ecological statistics," Iccv Vancouver:, 2001.

[28] C. Wei, W. Wang, W. Yang, and J. Liu, "Deep retinex decomposition for low-light enhancement," arXiv preprint arXiv:1808.04560, 2018. 